\title{
Oubliées ou disparues : Matérialiser absences et mémoires
}

Forgotten or missing: materializing absences and memories

Julie Graff

\section{(2) OpenEdition}

12 Journals

Édition électronique

URL : http://journals.openedition.org/iss/2101

DOI : $10.4000 /$ iss. 2101

ISSN : 2306-4161

Éditeur

ICOM - International Council of Museums

\section{Édition imprimée}

Date de publication : 1 août 2020

Pagination : 133-146

ISBN : 978-2-491997-11-3

ISSN : 2309-1290

Référence électronique

Julie Graff, «Oubliées ou disparues : Matérialiser absences et mémoires », ICOFOM Study Series [En ligne], 48-1 | 2020, mis en ligne le 01 août 2020, consulté le 21 décembre 2020. URL : http:// journals.openedition.org/iss/2101; DOI : https://doi.org/10.4000/iss.2101 


\section{Oubliées ou disparues : Matérialiser absences et mémoires}

\section{Julie Graff}

Université de Montréal, Montréal, Canada; EHESS, Paris, France

\section{Comment transcender l'oubli?}

L'art et ses multiples messages nous raménent toujours au brulant de nos organes, à l'évidence de nos chairs soudées. Avec pour armes les miracles de la douceur et de l'empathie nous avancerons dans cette trop longue histoire de déshumanisation qui

doit être ramenée aux fondamentaux de nos consciences.

Ces femmes, ce sont toutes les femmes du monde. Regardons leur histoire en face. Regardons notre histoire.

\section{Marie-Andrée Gill}

(Disponible sur le site internet de l'exposition oubliées ou disparues) 


\section{R'́SUMÉ}

L'exposition Öndia'tahterendih, oubliées ou disparues: Akonessen, Zitya, Marie et les autres, créée par la Boite Rouge Vif, une OBNL autochtone du Québec, et la commissaire wendate Sylvie Paré, présente 10 artistes rendant hommage aux femmes et aux filles autochtones touchées par le féminicide autochtone, tout en cherchant à mobiliser le public. Je souhaite dans le cadre de cet article explorer les stratégies mises en place au sein de l'exposition, telle qu'elle a été présentée au musée de la Civilisation du Québec en 2018. Je conclurai en explorant l'inscription de l'exposition dans le développement d'un commissariat engagé autochtone au Canada.

Mots clefs : muséologie autochtone, commissariat engagé, féminicide autochtone

\section{A B STRACT}

\section{Forgotten or missing: materializing absences and memories}

Öndia'tahterendih, forgotten or missing: Akonessen, Zitya, Marie and the others is an exhibition curated by the Boite Rouge Vif, an NPO from Quebec and wendat curator Sylvie Paré. The exhibition presents 10 artists honouring murdered and mission Indigenous women and girls, while seeking to emotionally engage the public with the issue of Indigenous feminicide in Canada. In this article, I would like to explore some of the curatorial strategies in this exhibition presented at musée de la Civilisation du Québec, in 2018, as part of an ongoing project engaging Indigenous curators in Canada.

Keywords: Indigenous museum practices, critical curating, Indigenous feminicide

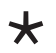

«Comment transcender l'oubli? » nous demande la poétesse Marie-Andrée Gill (Mashteuiatsh, nation ilnue) à l'entrée de l'exposition Öndia'tahterendih, oubliées ou disparues: Akonessen, Zitya, Marie et les autres (Oubliées ou disparues). Io artistes racontent, en faisant appel à une diversité de médiums et de techniques, la tragédie du féminicide autochtone au Canada, tout en rendant hommage à la vitalité culturelle des femmes autochtones. Créée par la Boite Rouge Vif, une OBNL (organisation à but non lucratif) autochtone du Québec qui développe des projets en transmission culturelle, l'exposition itinérante est présentée au cours de la deuxième moitié des années zoro dans une variété d'institutions 
culturelles : le centre d'art Langage Plus à Alma, la galerie Urban Shaman à Winnipeg, la maison de la culture Frontenac et l'espace Ashukan à Montréal/ Tiohtia :ke, ou encore le musée de la Civilisation à Québec (La Boite Rouge Vif, s. d.).

Les femmes, les filles et les personnes $2 E_{L G B T Q Q I A}{ }^{\mathrm{I}}$ membres des Premiers Peuples (Premières Nations, Métis, et Inuit) au Canada sont effectivement particulièrement touché.e.s par un ensemble de violences interpersonnelles, institutionnalisées et systémiques, enracinées dans une violence coloniale passée et actuelle. Plus particulièrement, leurs meurtres et leurs disparitions ont atteint une telle ampleur qu'ils ont été qualifiés d'épidémique par le rapporteur spécial des Nations Unies sur les droits des peuples autochtones, James Anaya (Enquête nationale sur les femmes et les filles autochtones disparues et assassinées, 2019). Au cours des 40 dernières années, plus de izoo femmes et filles autochtones ont été assassinées ou sont portées disparues au Canada². En 20IO, l'Association des femmes autochtones du Canada a ainsi confirmé l'existence de 582 dossiers de femmes et de filles autochtones disparues ou assassinées sur une période de plus de 20 ans. La Gendarmerie royale du Canada a alors dû mener sa propre enquête et constater II 8 I cas déclarés à la police entre I980 et 2012 (Enquête nationale sur les femmes et les filles autochtones disparues et assassinées, 2019).

Une Enquête nationale sur les femmes et les filles autochtones disparues et assassinées est finalement lancée en 2016 sous la pression militante pour se pencher sur les violences commises à l'encontre des femmes, filles, et personnes 2ELGBTQQIA. Dans son rapport final publié en 2019, l'Enquête nationale reconnaît autant la pluridimensionalité que l'ancrage colonial de ces violences :

Il n'existe pas une forme unique de violence contre les femmes autochtones ni une cause unique expliquant les disparitions et les meurtres de femmes et de filles autochtones. Réduire la violence à laquelle les femmes sont confrontées à sa seule forme interpersonnelle est une interprétation beaucoup trop limitée pour en comprendre la source. (...) Nous maintenons que la violence coloniale est complice de toute ces formes de violence. (p.351)

La commissaire et les créatrices ont alors au cœur de leurs préoccupations de présenter des œuvres s'éloignant d'un portrait statistique du féminicide, afin

\footnotetext{
I. 2ELGBTQQIA : Personnes bispirituelles, gays, lesbiennes, bisexuelles, transgenres, queer, en questionnement, intersexuées ou asexuelles (Enquête nationale sur les femmes et les filles autochtones disparues et assassinées, 2019).

2. Ce chiffre est le plus optimiste puisqu'il n'inclut pas plusieurs situations, comme les disparitions jamais reportées, les décès suspects, les disparitions et meurtres de personnes 2EGLBTQQIA (Ibid.)
} 
d'engager différemment le public dans leur compréhension de cette tragédie. Le commissariat de l'exposition, assuré par Sylvie Paré (Wendake, nation wendate), artiste et muséologue, répond aussi à des régimes d'historicité conventionnellement présentés dans les musées et touchant les femmes autochtones, leurs parcours de vie, leurs savoirs et savoir-faire. L'exposition lie ainsi leur perte aux pertes culturelles vécues par les communautés autochtones. Je souhaite alors dans le cadre de cet article explorer les stratégies mises en place au sein de l'exposition, telle qu'elle a été présentée au musée de la Civilisation du Québec du 23 mai 2018 au i7 février 2019 (musée de la Civilisation, 20I8). Je conclue en explorant l'inscription de l'exposition dans le développement d'un commissariat engagé autochtone au Canada. Les processus de décolonisation des institutions muséales ne pourraient effectivement pas exister sans le travail de praticien.ne.s et intellectuel.le.s autochtones. Ryan Rice, commissaire Kanien'kéha:ka, a ainsi décrit sa pratique de la façon suivante : «curator is also an architect, one who has the foresight to build an approachable, empathetic space that can broaden perspectives within the cultural landscape » (Rice, 2018, p. I06). Le commissariat autochtone peut alors se comprendre comme un processus de recouvrement d'une souveraineté culturelle autochtone qui passe par l'ouverture à de nouvelles perspectives. Oubliées ou Disparues renversent ainsi dynamiques d'exclusion et d'appropriation en remettant les personnes et leurs réalités au cœur des représentations et en réinscrivant les objets au sein d'une continuité culturelle impliquant agentivité et créativité (Isaac, 20I6; Rice, 20I8).

\section{Matérialiser l'absence}

Les œuvres présentées s'éloignent du portrait rationalisé et anonymisé des violences à l'encontre des femmes et filles autochtones en s'ancrant plutôt dans l'expression de la perte, de la souffrance et de la douleur vécues. Oubliées et Disparues, autant par les œuvres exposées que par sa construction expographique, performe un ensemble d'actions esthétiques structurant l'espace et le temps de la visite et propose au public une compréhension affective du drame, en matérialisant souffrance et absence. D'autres stratégies plus informatives, verbalement articulées, sont aussi mises en place. Elles fonctionnent autant comme des outils de contextualisation que comme des stratégies de renforcement de la présence des voix autochtones. Quelques dispositifs entourent à cet effet les ouvres, afin de présenter les créatrices et leurs perspectives sur leurs œuvres, par le biais de citations et de biographies, dont un long témoignage audio de l'artiste Diane Blacksmith (Mashteuiatsh, nation ilnue) sur son parcours de vie. Finalement, un documentaire produit par l'artiste multidisciplinaire Akienda Lainé (Wendake, nation wendate) présente les témoignages de plusieurs membres de communautés autochtones au Québec sur les relations hommes-femmes, leurs expériences et leurs espoirs pour le futur.

Nadia Myre (Kitigan Zibi, nation anishinabe) propose ainsi une production audiovisuelle, Untitled / Anonymes (2015), qui exprime, par la vidéo d'un long 
passage dans un tunnel obscur se terminant par une course désorientée dans un décor sylvestre, l'anxiété et la frayeur des femmes victimisées :

Une femme court la nuit, sur une route parmi les ombres. Ces femmes, aux prises avec des situations terribles, cherchent à s'échapper et à trouver un monde meilleur. Trop souvent, elles se retrouvent face à un mur, celui de la violence quelles cherchent à fuir.

\section{9}

La mise en exposition, prenant tout un pan de mur dans un espace de visionnement installé au milieu de la salle et coupé du reste de l'exposition, accentue le point de vue narratif interne adopté par la vidéo. L'ouvre plonge alors la visiteuse ou le visiteur dans une expérience haptique, en jouant sur le sentiment d'identification.

Deux œuvres matérialisent plus particulièrement l'absence, en reconstituant les espaces laissés vides par la disparition. Ainsi Mariette Manigouche (Mashteuiatsh, nation ilnue) constitue un autel, Petit baluchon et mitaines (2015), sur lequel se trouvent plusieurs objets, baluchons et mitaines en peau d'orignal, tresses de foins d'odeurs, entourant la reproduction d'une ouvre de William Armstrong, Portrait of an Indian Woman (I86I). L'autel est protégé par un demicercle formé de pierres rondes. Mariette Manigouche dédie alors cet autel aux femmes disparues: "Les chambres des femmes disparues sont des autels où chaque petit objet rappelle cette femme qui est toujours importante pour quelqu'un. » L'exposition propose aussi une œuvre collective, effort conjoint des artistes et de l'équipe commissariale. Cette installation, Chambre d'une jeune fille disparue (2017), adaptée in situ, reconstitue une chambre d'adolescente, avec ses meubles, décorations, un ordinateur, un miroir et son maquillage, des vêtements éparpillés, un cactus en pot... et aussi un ensemble de broderie et de perlage, une paire de mocassin, un attrape-rêve. Le tout est accompagné d'un ensemble d'objets issus des collections du musée de la Civilisation leur faisant écho, par leurs fonctions, techniques ou motifs. Les visiteur.se.s sont alors confronté.e.s à la présence accablante et désespérante de l'absence en étant placé.e.s devant un espace très familier, la chambre :

Cette chambre est celle de toutes les jeunes filles autochtones, les objets qu'elles aiment, qui témoignent de leur vie entre deux cultures. Pour les proches, c'est la tragédie de se retrouver avec une chambre vide du jour au lendemain en espérant leur retour.

\footnotetext{
I. Les collaboratrices sont Sylvie Paré, Diane Blacksmith, Lise Bibeau, Jocelyn Fortin, Milène Essertaize, Mélissa Corbeil, Claudia Néron, Justine Bourdages et Camille Perry.
} 
Cette approche, à l'égard d'autres stratégies artistiques explorées par les créatrices, se rapproche de l'idée d'aesthetic action (action esthétique), développée par Robinson et Martin (2016). Ce concept a tout d'abord permis de qualifier l'ensemble des stratégies esthétiques mises en place durant la Commission de vérité et réconciliation du Canada (2008-2015) cherchant à faire la lumière sur les pensionnats indiens et les traumatismes qu'ils ont causés au sein des communautés (Moran, 2015). L'émotion forte d'une souffrance multigénérationnelle avait alors trouvé une partie de son expression dans un ensemble d'actes et de productions esthétiques. Pour Robinson et Martin, ces actions esthétiques englobent un vaste ensemble de productions: « a range of sensory stimuli-image, sound and movement- [that] have social and political effects through our affective engagements with them » (Robinson \& Martin, 2016, p. 2). Ces expériences possèdent ainsi une agentivité reposant sur un apprentissage incorporé, c'est-à-dire intégrant l'ensemble des sensations et des émotions informant notre compréhension du monde, un mode d'apprentissage et de compréhension dont l'impact est souvent sous-estimé. L'empathie demandée des visiteur.se.s a alors le potentiel de les engager dans une responsabilité sociale de conscientisation et de mobilisation. Ainsi il est aussi proposé au public, directement au sein de l'exposition, de signer une pétition de soutien les engageant «à faire preuve de solidarité envers les femmes autochtones disparues ou oubliées et leurs proches. »

Cette souffrance touche ainsi non seulement les femmes, mais aussi leur famille et leur communauté. Hannah Claus (Baie de Quinte, nation kanien'keha :ka) exprime de ce fait la relationalité de la perte, avec une ouvre honorant les femmes disparues tout en marquant les bouleversements provoqués par leur absence. Hannah Claus utilise une liste de noms, complétée d'après les bases de données des forces policières de l'ensemble du pays, qui s'affichent les uns après les autres sur une projection. Celle-ci est entourée d'un ensemble de petites maquettes de maisons en tuile d'aluminium dont certaines sont renversées, formant alors les strates de souffrance touchant les individus, et au-delà leurs familles et leurs communautés. La projection fut réalisée avec la collaboration de volontaires provenant des communautés d'Odanak, de Kahnawà :ke, de Kanehsatà :ké, du collège Kiuna et de l'Université Concordia. For those who didn't make it home/ pour celles qui ne sont jamais revenues (2015) se construit ainsi par la mobilisation d'une communauté de soutien grâce à un ensemble d'actes de commémoration. L'efficacité de l'œuvre prend alors place à la fois dans son temps de production et dans son temps d'exposition, explorant ainsi le potentiel de la création artistique comme outil de transfert du pouvoir à l'audience, par la collaboration dans un premier temps et l'engagement sensible avec l'œuvre par la suite (McCall \& L'Hirondelle Hill, 20I5). Claus met ainsi de l'avant la relationalité, les réseaux de relations, familiaux et communautaires, dans lesquels s'inscrivent les femmes disparues. 


\section{Combattre l'oubli}

Au-delà de l'absence, l'exposition porte un geste contre une strate supplémentaire de violence à l'encontre des femmes et des filles autochtones : l'oubli, sous ses différentes formes. La violence dénoncée est historique autant qu'actuelle; elle est non seulement physique et psychologique, mais aussi mémorielle : oubli des noms et des savoirs, invisibilisation des individus et des histoires. Hannah Claus centre ainsi son œuvre autour d'un travail collectif de mémoire des noms, des identités. D'autres artistes cherchent aussi à réinscrire ce travail de mémoire dans le temps, en dénonçant des stratégies historiques d'invisibilisation ayant contribué à la marginalisation à laquelle font face les communautés autochtones au Canada. Effectivement, la représentation des peuples autochtones fut marquée, particulièrement à la fin du Ig ${ }^{\text {ème }}$ siècle et au début du $20^{\text {ème }}$ siècle, par l'idée persistante des formes sociales non-occidentales disparaissant au moment même où elles sont représentées par un travail ethnographique ou littéraire (ou muséographique) (Clifford, I988). Deborah Doxtator, historienne et muséologue Kanien'kehá:ka, démontre l'inscription de ces stéréotypes et de leurs expressions culturelles dans les dispositifs de contrôle et de dépossession des peuples autochtones, dont la représentation échappe à leur contrôle: "They are designed to influence not only how society views certain groups, but also attempt to control how people see themselves » (Doxtator, I992, p. I4). Ce paradigme a justifié une collecte sauvage de restes humains, d'artefacts, d'objets funéraires, de pratiques culturelles, de connaissances, de mémoires, sans aucun contrôle de la part des autochtones par la suite sur leur usage, leur présentation et leur circulation dans le monde muséal, scientifique et culturel. Leur présupposée disparition facilite cette appropriation, et aboutit à une véritable invisibilisation institutionnelle des individus, au profit de la construction visuelle d'une altérité spécifique (Clifford, I988; Doxtator, 1996). Ces paradigmes historiques, particulièrement ceux enracinés dans le déni de l'historicité et de la contemporanéité des peuples autochtones, sont de ce fait un premier enjeu dans le projet critique de réécriture historiographique mené par des intellectuel.le.s autochtones (H. L. Igloliorte, 2012). Ainsi, Oubliées ou Disparues intègre les ouvres de Diane Robertson (I960-I993; Mashteuiatsh, nation ilnue), artiste contemporaine autochtone pionnière. Les études de corps présentées s'inscrivent dans une carrière prolifique prenant place dans les années I980 et coupée subitement à la mort de l'artiste. Ces ouvres sont alors, à l'image de plusieurs ouvres de l'exposition, témoins de l'engagement continu des artistes dans de nouveaux médiums et des nouvelles filiations qui se construisent. Elles démentent alors le déni de contemporaneité auquel a dû faire face la création autochtone, enfermée jusqu'à récemment dans une vision objectifiante et passéiste aux dépens de la reconnaissance de la vitalité et de l'innovation artistiques (Uzel, 20I7).

Une installation par une artiste anonyme s'attarde ainsi sur les liens de causalité qui peuvent être tissées entre invisibilité institutionnelle des femmes autochtones et leur victimisation. Disparition institutionalisée (2015) est constituée d'une 
série de reproductions de tableaux d'artistes allochtones du I $9^{\text {ème }}$ et début $20^{\text {ème }}$ siècle représentant des femmes autochtones et affublés de titres généralement génériques (Jeune Indienne, ou encore Veuve Indienne ${ }^{\mathrm{T}}$ ). Ces reproductions sont autant de témoins d'une histoire dans l'art de l'aliénation des corps, de l'absence des voix et de la suppression des identités. La commissaire Sylvie Paré exprime ainsi cette aliénation amnésique :

En amorçant ma recherche pour cette exposition, j'ai constaté combien les termes génériques comblent les vides laissés par l'histoire. Une succession infinie de titres tels que L'Indienne, Portrait d'une Indienne, Les jeunes Indiennes... défilent sur les fiches. À défaut d'en savoir plus sur ces femmes, les espaces blancs jouent pleinement leur rôle : celui de contraindre à la non-existence la contribution monumentale des femmes autochtones à l'histoire des Amériques.

Dans Disparition institutionalisée, les reproductions d'œuvres surplombent une série de crochet reproduisant un mur de vestiaire. Des petites plaques de cuivre au-dessus de chaque crochet offrent le nom d'une femme victimisée. Sous le nom de Loretta, un sac de sport sale est suspendu. Un texte, exposé au milieu des reproductions d'œuvres, fait froid dans le dos par la compréhension qu'il offre de ce sac. Il s'inspire de l'ouvrage Soeurs volées, enquête sur un féminicide au Canada, d'Emmanuelle Walter (20I4) :

Moi, Loretta, javais 26 ans et j'étais enceinte depuis trois mois. J'étais Inuk (Inuite) et j'étudiais à l'Université St-Mary, à Halifax, à 2000 kilométres de ma petite ville natale du Labrador. J'écrivais une thèse sur les femmes autochtones disparues et assassinées.

J'ai été tuée, pense la police, le 13 février, soit le jour de la remise d'une pétition de 23 ooo signatures réclamant une enquête nationale, et la veille des marches commémoratives annuelles à travers le pays.

[...] mon corps a été retrouvé au Nouveau-Brunswick treize jours après ma disparition dans un sac de hockey couvert de neige, au bord de l'autoroute transcanadienne. 
L'oubli peut ainsi se cacher derrière les représentations, particulièrement les représentations aliénantes des femmes autochtones. Partie intégrante de l'histoire coloniale du Canada, elles impliquent l'oubli du nom, de l'individu, au profit d'archétypes. Ces conceptions historiques déshumanisent les individus, légitiment la violence coloniale, informent et accompagnent les représentations contemporaines, principalement les représentations médiatiques ayant souvent traité les disparitions et les meurtres des femmes et filles autochtones avec laxisme (Enquête nationale sur les femmes et les filles autochtones disparues et assassinées, 2019). Une invisibilisation dénoncée par Marie-Andrée Gill dans son texte d'introduction à l'exposition, où elle lie invisibilisation et fragilisation :

L'image de la femme autochtone telle qu'inventée par la colonisation est restée accrochée aux têtes, comme tous les clichés de la méconnaissance. Femmes libres, femmes aimantes, enveloppent de leur chaleur des millénaires de culture et de soins, étaient vues comme simplement belles et dociles, sans foi ni loi : elles ont été désincarnées.

Cette image objectivée est restée, et a abouti à une inévitabilité: la fragilisation. De ces constats historiques est resté ce qu'on voit aujourd'hui, un désastre familial et collectif: les mères, les amies, les soeurs, Aknessen, Zitya, Tina, Marie... oubliées ou disparues.

\section{Célébrer les matrimoines}

L'artiste Sylvie Bernard (Wôlinak, nation abénakise) s'engage ainsi dans la commémoration de femmes de sa communauté qui furent traitées par cette amnésie historienne. Ses deux ouvres présentées, Vêtement - sculpture portable et Croix - sculpture portable, font partie d'une série de 6o sculptures portables dédiées à 60 femmes sans noms. La série Les noms-dits est ainsi partie de l'étude par l'artiste des Actes de sépultures des Abénakis de Wôlinak (1719-1899), souvent seule trace restante de l'existence des membres de sa communauté. Cherchant alors à réécrire l'histoire de ces 60 femmes inhumées sans nom, Sylvie Bernard « tisse l'oubli » dans des sculptures qui font appel au savoir dont elle se perçoit comme l'héritière et qui la lie dans la connaissance et la matérialité aux femmes des décennies et siècles précédents.

Cette continuité des savoirs informe l'ensemble de l'exposition. Effectivement, tel qu'il est souligné non seulement dans l'exposition mais aussi dans le rapport de l'Enquête Nationale sur les femmes et les filles disparues et assassinées, la perte des savoirs et des savoir-faire s'inscrit dans les violences coloniales, passées et actuelles, menaçant la sécurité culturelle des femmes et des filles autochtones en portant atteinte à leur droit à la culture et à leur capacité de pratiquer et de transmettre (Enquête nationale sur les femmes et les filles autochtones disparues et assassinées, 2019). Un exemple frappant, parmi d'autres, de cette 
violence est l'interdiction des pratiques et du port de vêtements marquant l'identité culturelle par la Loi sur les Indiens entre i8 86 et 195I(Henderson, 20I8). L'exposition explore ainsi les enjeux reliés à la transmission culturelle par la célébration des mémoires et des matrimoines technologiques des Premiers Peuples. Les œuvres sélectionnées vont de la vannerie au film et toutefois se répondent l'une à l'autre en explorant des expériences partagées de souffrance et de survivance. Leur assemblage illustre ce désintérêt pour marquer des catégories, que ce soit entre art et artisanat ou entre tradition et innovation, un désintérêt renforcé par la diversité des espaces ayant accueilli l'exposition. La continuité culturelle ainsi revendiquée permet de construire un régime particulier d'historicité, défini par Heather Igloliorte et Carla Taunton comme : «a diachronic project of linking past and present. » (2017, p. 5). Ainsi, Lise Bibeau et Annette Nolett (Odanak, nation abénakise) proposent un hommage aux générations précédentes :

Hommage aux femmes abénakises, nos mére et nos grands-méres qui ont assuré la survie de leurs familles pendant plus d'un siècle en maniant de leurs mains agiles le frêne et le foin d'odeur. Nous leur offrons ce cèdre, don de notre Terre-Mére.

Wanilijhsjik Phanemok (2015) est un panier tissé en frêne noir, en cordes de coton et en foin d'odeur, rempli de branches de cèdre, en don aux générations les ayant précédés et ayant assuré la transmission des techniques utilisées. Diane Blacksmith propose une réflexion similaire avec l'installation Châle de Kukum (2015), qui met en valeur un vêtement multigénérationnel et les savoirs derrière sa production :

Des générations de femmes ilnues ont porté le châle en tissus écossais. J'ai tanné et travaillé le cuir pour en fabriquer un en hommage à l'amie que j'ai perdue. Je l'ai fait en son honneur, pour elle, pour nos grands-méres et pour les femmes disparues.

9

Le châle est suspendu à une grande branche de bouleau qui a l'air de jaillir d'un coin de sa salle. S'inscrivant dans une mémoire matrimoniale, vivante et innovante, Blacksmith peut ainsi porter hommage par le geste de création et par l'acte d'exposition.

\section{En guise de conclusion : transformer les espaces}

La vitalité culturelle et la réécriture historiographique sont ainsi deux enjeux qui touchent non seulement la production artistique mais aussi, et peut-être 
surtout, son exposition, d'où l'importance du travail mené par les commissaires autochtones dans leurs pratiques et leurs écrits. Doxtator réclame ainsi une réécriture historiographique par les Autochtones eux-mêmes afin de se réapproprier l'autorité sur leur passé : une forme de propriété qui en appelle à la responsabilité de chacun, ce qu'elle exprime par la plurivocalité du verbe 'to own' :

\begin{abstract}
Aboriginal peoples in Canada over the past decade have experienced an increasing need to understand and, once again, to own our cultural past, present, and future. I've been thinking a lot about the verb 'to own'. It boils down to a notion of owning as property, but that's not really all the word means. It's certainly not what I mean. I'm talking about owning who we are (...) What I mean is that you own the responsability of who you are and what you belong to. It's a slow realization that you have a responsibility to participate, and that this is the responsibility given to you as an aboriginal person
\end{abstract}

(Doxtator, I996, p. 56).

\title{
99
}

Le Canada, dans la deuxième moitié du vingtième siècle, et plus particulièrement à partir des années I990, a vu l'émergence et la consolidation d'un mouvement de renégociation des relations entre musées et peuples autochtones. Plusieurs décennies de revendications des droits des peuples autochtones ont effectivement rendu possible le développement d'un corpus rhétorique et visuel défendant la souveraineté autochtone afin de restaurer l'autonomie culturelle, intellectuelle et politique de ces peuples. Ce projet de restauration constitue ainsi un vaste mouvement de décolonisation, mis en place par les communautés autochtones, et touchant toutes les institutions ayant un impact sur leur vie et leurs représentations (Smith I999; Alfred, 2008; Clifford, 2013). Le monde du pa/matrimoine et des musées a ainsi dû accepter ses responsabilités pour le rôle joué par les institutions muséales dans la colonisation et l'assimilation forcée des peuples autochtones (Phillips, 20II). De ce fait, le déni d'une contemporanéité de la création autochtone, de toute forme d'historicité en fait, a poussé les artistes autochtones à prendre en main et créer par eux-mêmes des opportunités de diffusion, en se faisant galeristes et commissaires. Ainsi, en I962, Doug Cranmer, artiste et sculpteur kwakwakawa'k, ouvre à Winnipeg la Talking Stick Gallery afin d'avoir la possibilité de s'exposer. Cette galerie ferme malheureusement ses portes en I967, mais quelques années plus tard c'est au tour de Daphne Odjig, artiste anishinabe, d'être exacerbée de la réception qu'elle rencontre dans le monde de l'art. La New Warehouse Gallery, fondée en I97I, devient ainsi un espace central de représentation pour les artistes contemporains autochtones, un lieu de rassemblement et d'échange essentiel à l'inclusion et la diffusion des artistes. Deux ans plus tard, Daphne Odjig s'associe avec Norval Morisseau pour fonder la Professional Native Indian Artists Incorporation, 
qui répond alors au besoin d'un réseau de soutien et d'information des artistes autochtones contemporains (Isaac, 20I6; Logan, 2004; Martin, 2004). Plusieurs générations de praticien.ne.s vont ainsi marquer leur présence non seulement en tant qu'artistes, mais aussi en tant que théoricien.ne.s et commissaires, favorisant les carrières de leurs collègues. Joane Cardinal-Schubert, artiste kainaiwa et commissaire, témoigne ainsi sur les années I970 et 1980 :

As artists, we have kicked down doors having to assume dual roles of curators and historians lobbying with governments, educational institutions, funding agencies, galleries and even our relatives, friends and peers. Some of us never returned to being full-time artists, remaining locked in a needed service for our own people

(Cardinal-Schubert, 2004).

9

Sylvie Paré est elle-même artiste et muséologue et travaille, entre autres, pour le Jardin des Premières Nations au Jardin Botanique de Montréal. Elle exprime aussi ce besoin de faire acte de présence pour d'autres artistes autochtones :

Souvent les conservateurs ou les conservatrices [autochtones] sont des artistes. Ils ont fait un cheminement un peu comme moi (...) Le fait qu'il y a eu tellement de portes à ouvrir, et souvent les portes n'étaient pas ouvertes, à un moment donné tu as envie de donner à ceux qui te suivent, en quelque sorte aux autres générations. Tu te dis : "(...) Je suis aussi bien de moccuper de la génération qui s'en vient, et de voir ce qu'on peut faire ensemble, peut-être aurons-nous plus de poids. " (2018) ${ }^{1}$

Malgré ces portes enfoncées au cours des 6o dernières années, d'importants défis demeurent encore aujourd'hui, que ce soit dans les formations offertes, les opportunités professionnelles ou encore le développement de projets dans diverses institutions (Isaac, 2016). L'inclusion même de praticiens autochtones dans les institutions problématise le potentiel du musée comme un espace d'accueil viable (Tanguay \& Kaine, 20I8). Un commissariat qui s'identifie comme un commissariat engagé autochtone va alors tenter de transformer les modalités discursives et expographiques qui canalisent la diffusion de l'art contemporain, en espérant que l'engagement des institutions et du public perdure au-delà de l'exposition.

I. Entretien avec l'auteure, 25 septembre 2018. 


\section{Références}

Alfred, T. (2008). Peace, Power, Righteousness : An Indigenous Manifesto (2 édition). Don Mills; New York: Oxford University Press.

Cardinal-Schubert, J. (2004). Flying with Louis. Dans L.-A. Martin (Éd.), Making a noise! Aboriginal perspectives on art, art history, critical writing, and community (p. 26-49). Banff: Banff Centre Press.

Clifford, J. (I988). The predicament of culture: Twentieth-century ethnography, literature, and art. Cambridge, Mass: Harvard University Press.

Clifford, J. (2013). Returns: Becoming indigenous in the twenty-first century. Cambridge, Massachusetts: Harvard University Press.

Doxtator, D. (1992). Fluffs and feathers: An exhibit on the symbols of Indianness. A resource guide. (Rev. ed). Brantford, Ontario: Woodland Cultural Centre.

Doxtator, D. (1996). The implications of Canadian nationalism for Aboriginal cultural autonomy. In Curatorship : Indigenous perspectives in post-colonial societies: proceedings (p. 56-76). Ottawa: Canadian Museum of Civilization; Commonwealth Association of Museums; University of Victoria.

Enquête nationale sur les femmes et les filles autochtones disparues et assassinées. (2019). Réclamer notre pouvoir et notre place: Le rapport final de l'enquête nationale sur les femmes et les filles autochtones disparues et assassinées. Page consulté le I6 novembre 2019, https://www.mmiwg-ffada. $\mathrm{ca} / \mathrm{fr} /$ final-report/

Henderson, W. B. (2018). Loi sur les Indiens. In The Canadian Encyclopedia. Page consulté le i6 novembre 20I9, https://www.thecanadianencyclopedia. $\mathrm{ca} / \mathrm{fr} /$ article/loi-sur-les-indiens

Igloliorte, H. (2012). « Pas de colonialisme dans notre histoire. » Les pratiques de décolonisation dans l'art indigène. Dans H. Igloliorte, B. L. Croft, \& S. Loft (Eds.), Decolonize me/ Décolonisez-moi (p. 28-37). Ottawa: Ottawa Art Gallery.

Igloliorte, H., \& Taunton, C. (20I7). Introduction : continuities between eras: Indigenous Art histories / Introduction : continuité entre les époques : histoires des arts autochtones. RACAR, 42(2), 5-I2.

Isaac, J. L. (2016). Decolonizing curatorial practice : acknowledging Indigenous curatorial craxis, mapping its agency, recognizing its aesthetic within contemporary Canadian Art. Mémoire. University of British Columbia, Vancouver.

La Boite Rouge Vif. (s. d.). Oubliées ou disparues. Page consultée le i6 novembre 20I9, http://oublieesoudisparues.ca/wp/ 
Logan, J. (2004). It's not just noise. Dans L.-A. Martin (Éd.), Making a noise! Aboriginal perspectives on art, art history, critical writing, and community (p. 72-82). Banff: Banff Centre Press.

Martin, L.-A. (2004). Making a Noise in this (Art) World! Dans L.-A. Martin (Éd.), Making a noise! Aboriginal perspectives on art, art history, critical writing, and community (p. I6-23). Banff: Banff Centre Press.

McCall, S., \& L'Hirondelle Hill, G. (Eds.). (2015). The land we are: Artists and writers unsettle the politics of reconciliation. Winnipeg: ARP Books.

Moran, R. (2015). Commission de vérité et réconciliation du Canada. In L'Encyclopédie Canadienne. Page consulté le I6 novembre 2019, https://www. thecanadianencyclopedia.ca/fr/article/commission-de-verite-et-reconciliation-du-canada

Musée de la Civilisation. (2018, mai 22). Oubliées ou disparues: Akonessen, Zitya, Tina, Marie et les autres. Musée de la Civilisation de Québec. Page consulté le I6 novembre 20I9, https://www.mcq.org/fr/communique-presse?id=74I900

Phillips, R. B. (20II). Museum Pieces: Toward the Indigenization of Canadian Museums. Montreal: McGill-Queen's University Press.

Réseau pour la stratégie urbaine de la communauté autochtone à Montréal. (2019). Trousse d'outils pour les alliées aux luttes autochtones. Page consulté le I6 novembre 2org https://gallery.mailchimp.com/86d28ccd $43 \mathrm{~d}_{4}$ beocfспіс7таг/files/84889i8o-9bfo-46f2-8deo-dc932e4850i3/FR_Ally_email. pdf

Rice, R. (20I8). Curator's Statements / Réflexions des commissaires. RACAR, $43(2)$, Io6.

Robinson, D., \& Martin, K. (2016). The Body is a Resonant Chamber. In D. Robinson \& K. Martin (Eds.), Arts of engagement : taking aesthetic action in and beyond the Truth and Reconciliation Commission of Canada (p. I-20). Waterloo, Canada: Wilfrid Laurier University Press.

Smith, L. T. (I999). Decolonizing methodologies: research and Indigenous peoples. London: Zed Books.

Tanguay, J., \& Kaine, É. (20I8). Créativité et muséologie autochtone: Dialogue entre conservateurs-artistes autochtones d'Australie et du Québec. Recherches amérindiennes au Québec, 48(I-2), I75-I8I.

Uzel, J.-P. (20I7). Déni et ignorance de l'historicité autochtone dans l'histoire de l'art occidentale. RACAR, $4^{2}(7), 30-4 \mathrm{I}$.

Walter, E. (20I4). Sours volées: Enquête sur un féminicide au Canada. Montréal, Qc: Lux Éditeur. 